\title{
Variable Wave Plate via Tunable Form-Birefringent Structures
}

\author{
Benjamin K. Wilson and Lih Y. Lin, Senior Member, IEEE
}

\begin{abstract}
We propose a compact and low-cost design for a variable wave plate using tunable form-birefringent grating structures in polydimethylsiloxane (PDMS). The described device operates through PDMS deformation via electrostatically actuated transparent electrodes. The optical and mechanical properties of tunable form-birefringent structures are modeled using birefringence, rigorous coupled-wave analysis, and simple structural mechanics theory. The device is fabricated by a form of soft nanoimprint lithography. The optical properties are characterized for different structure geometries. Operating principles were verified through testing of the completed device. The experimental results and theoretical study show that an irregular grating structure performs better than a periodic subwavelength structure.

[2007-0303]
\end{abstract}

Index Terms-Form birefringence, nanoimprint lithography (NIL), optical polarization, tunable wave plate.

\section{INTRODUCTION}

$\mathbf{T}$ UNABLE WAVE plates are important components for applications and experiments in optics. They are also the building blocks for polarization controllers. Several models and studies of variable and static wave plates based on formbirefringent grating structures have been performed [1]-[4]. The chief challenge for implementing such devices is the need for a long path length (deep grating features) and a high index of refraction material required for the grating. Furthermore, a simple and low-cost way of tuning the grating structure to achieve a variable wave plate has not yet been demonstrated.

Form-birefringent wave plates have been demonstrated using several fabrication techniques and materials, such as ZTO [2], $\mathrm{TiO}_{2}$ [3], and GaAs [4]. While these materials can be used to achieve static wave plates, they do not easily lend themselves to being tunable because they are not actuable or optically active.

In recent years, several studies have been performed on the use of elastomers, including polydimethylsiloxane (PDMS), both as actuators and flexible gratings [5]-[7]. Perhaps the most common form of elastomer-based actuator is the dielectric elastomer actuator (DEA) [8]. Such an actuator operates by applying a voltage across a flexible dielectric material using

Manuscript received December 20, 2007; revised May 27, 2008. Published August 1, 2008 (projected). This work was supported in part by the National Science Foundation under Grant DBI-0454324 and in part by the National Institutes of Health under Grant 1-R21-EB005183. Subject Editor S. Merlo.

The authors are with the Department of Electrical Engineering, University of Washington, Seattle, WA 98195 USA (e-mail: bewilson@u.washington.edu; lylin@u.washington.edu).

Color versions of one or more of the figures in this paper are available online at http://ieeexplore.ieee.org.

Digital Object Identifier 10.1109/JMEMS.2008.927744

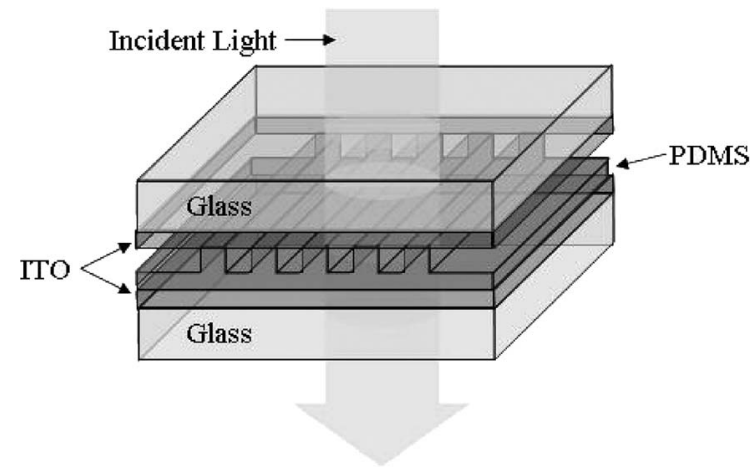

Fig. 1. Schematic of tunable form-birefringent wave plate.

a compliant electrode. Under the electrostatic attraction, the electrodes are drawn together, causing the elastomer and the electrode to expand outward in the plane of the electrode. DEAs have been shown to achieve strains greater than $100 \%$. Silicones such as PDMS have been used in DEAs with good results [9]. Such devices achieve large deformations with reasonable applied voltages. However, these devices are generally difficult to integrate with other micro-nano device technologies because they require compliant electrodes, which are difficult to integrate with other structures or devices.

In addition to their use as actuators, elastomers such as PDMS have also been used for making deformable optical devices such as diffraction gratings and lenses [5], [6], [10]-[12]. Deformable grating devices have been hindered by low degrees of tunability and slow response times. This can be attributed to the fact that they are generally made from large blocks of PDMS which require large forces to deform.

Using form-birefringent structures to realize a tunable wave plate presents several advantages over technologies such as $\mathrm{LiNbO}_{3}$ and liquid crystal. First, the primary material and fabrication process, PDMS and soft lithography, respectively, are low cost and capable of high-throughput fabrication. Furthermore, form-birefringent structures also show the potential for lower operating voltages and optical loss, both of which are advantageous for use in optical systems. Our proposed design for this tunable wave plate is shown in Fig. 1. The PDMS grating structure is compressed under electrostatic actuation, thus changing the fill factor which affects the phase difference between polarizations that are parallel and perpendicular to the grating pattern.

In this paper, we present design, theoretical modeling, and demonstration of the operating principle of a tunable wave plate made of deformable PDMS form-birefringent structure using nanoimprint lithography (NIL). 


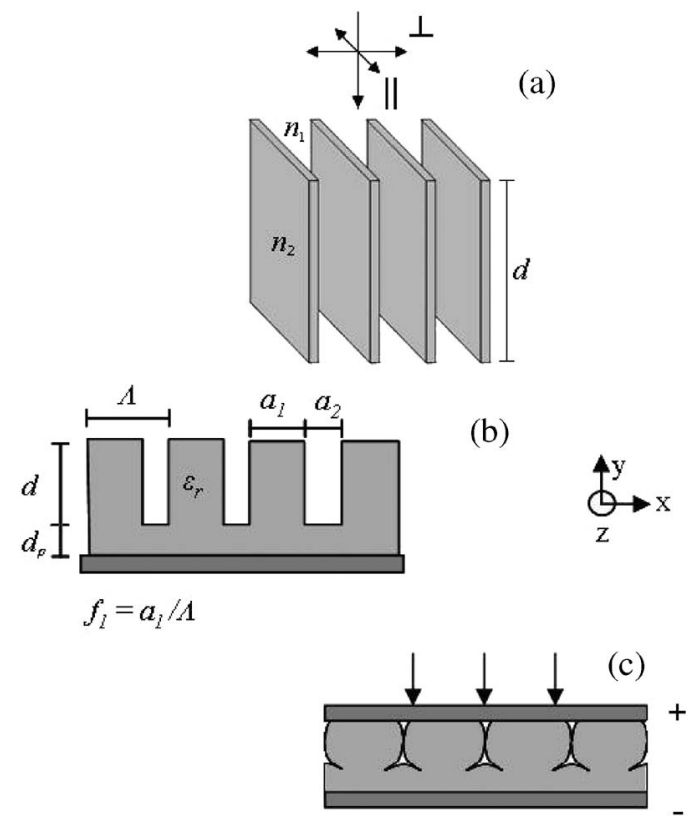

Fig. 2. Schematic representation of (a) parallel $(\|)$ and perpendicular $(\perp)$ polarization modes incident on an array of dielectric plates, (b) deformable PDMS structure, and (c) PDMS structure under compression.

\section{THEORY AND MOdeling}

\section{A. Optical Properties}

Birefringence is a common material property that describes how the index of refraction in certain materials is dependent on polarization and crystal orientation. Nearly all wave plates use this phenomenon to achieve phase delay between different polarizations. Form birefringence is the same property, which is only caused by the shape of the material and not the crystalline or molecular orientation. Form birefringence can be modeled using the method presented by Born and Wolf [13]. This simplified method describes the difference in effective refractive index seen by parallel and perpendicular polarization modes incident on a series of parallel dielectric plates, as shown in Fig. 2(a).

If the period of the array of dielectric plates is less than the wavelength, the effective index of refraction for each polarization mode can be assumed to be the average over the structure. These will be slightly different for the two polarization modes as defined in the following:

$$
\begin{aligned}
& n_{\|}=\sqrt{f_{1} n_{1}^{2}+f_{2} n_{2}^{2}} \\
& n_{\perp}=\sqrt{\frac{n_{1}^{2} n_{2}^{2}}{f_{1} n_{2}^{2}+f_{2} n_{1}^{2}}}
\end{aligned}
$$

where $f_{1}$ and $f_{2}$ represent the fill factors of the two media $\left(f_{1}+f_{2}=1\right)$ and $n_{1}$ and $n_{2}$ represent the indices of refraction of the two media, as shown in Fig. 2. The polarization phase delay therefore becomes a function of the fill factor, path length or depth of the material $(d)$, and index of refraction of the dielectric material. Fig. 3 shows the calculation result on phase delay versus different variables. All models are for structures in air $\left(n_{1}=1\right)$. In order to maximize polarization phase delay, a form-birefringent structure should be made from a high-

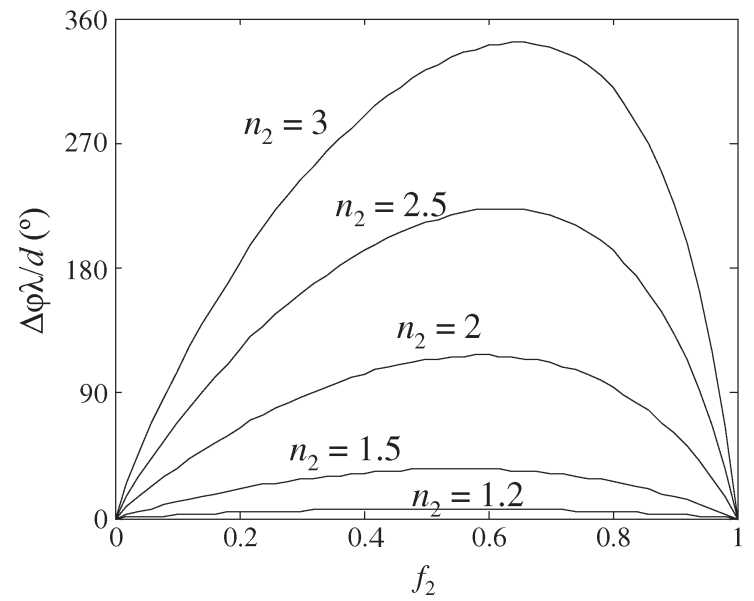

Fig. 3. Polarization phase delay $(\Delta \varphi \lambda / d)$ normalized to wavelength and depth versus dielectric fill factor for several dielectric indices of refraction.

index material, have deep dielectric structures compared to the wavelength, and have a fill factor of about $0.5-0.65$ (see Fig. 3).

As mentioned earlier, form-birefringence theory is based on the assumption that the period of the involved dielectric structures is much shorter than the wavelength of light [13]. However, such a structure with a period that is short compared to the wavelength and having sufficient depth is more challenging to fabricate using PDMS NIL. Therefore, it becomes necessary to use longer period structures and more complex electromagnetic-wave models which incorporate the effects of diffraction. Perhaps the most widely used model is rigorous coupled-wave analysis (RCWA) [14], [15]. RCWA uses coupled-wave equations for the harmonic modes of a propagating wave in a periodic structure matched with the appropriate boundary conditions to produce eigenvalue solutions to Maxwell's equations. The eigenvalues can be used to obtain information on transmission and reflection efficiencies as well as phase delay.

The effect of period on the performance of our formbirefringent structures as determined by RCWA can be observed in Fig. 4, which shows $\Delta \varphi$ and the transmission ratio for TE and TM polarization states for the zeroth-order mode $\left(D_{0}^{\mathrm{TE}} / D_{0}^{\mathrm{TM}}\right)$. Light was assumed to have an angle of incidence normal to the structure surface. When the period is less than the wavelength (i.e., $\Lambda / \lambda=0.5$ ), the structures act according to form-birefringence theory, namely, a $\Delta \varphi$ versus $d$ and $f$ relationship similar to form birefringence and very little variance between $D_{0}^{\mathrm{TE}}$ and $D_{0}^{\mathrm{TM}}\left(D_{0}^{\mathrm{TE}} / D_{0}^{\mathrm{TM}}=0 \mathrm{~dB}\right)$. However, when the period increases to the wavelength and beyond, form-birefringence theory quickly breaks down with much less predictable $\Delta \varphi$ versus $d$ and $f$ relationships and significant variance between $D_{0}^{\mathrm{TE}}$ and $D_{0}^{\mathrm{TM}}$ (hereafter called transmission-efficiency variance).

While the increased period leads to more unpredictable results, it is noted that the overall achievable polarization phase delay increases as well. To observe the effects of period on overall performance, a Jones matrix model can be made to account for both polarization phase delay and transmissionefficiency variance. Using this model, the effect of compressing the structures on the output polarization state can be plotted 

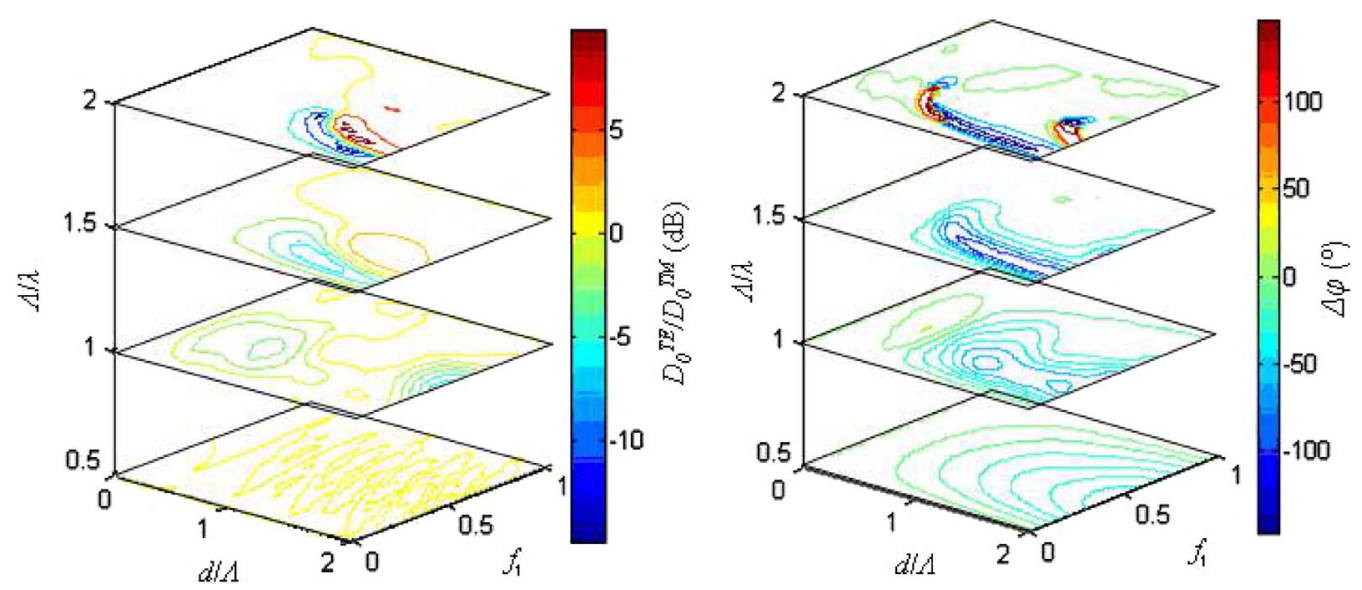

Fig. 4. Lines of (left) equal transmission-efficiency variance and (right) polarization phase-delay versus normalized depth, dielectric fill factor, and normalized period. $n=1.49$.

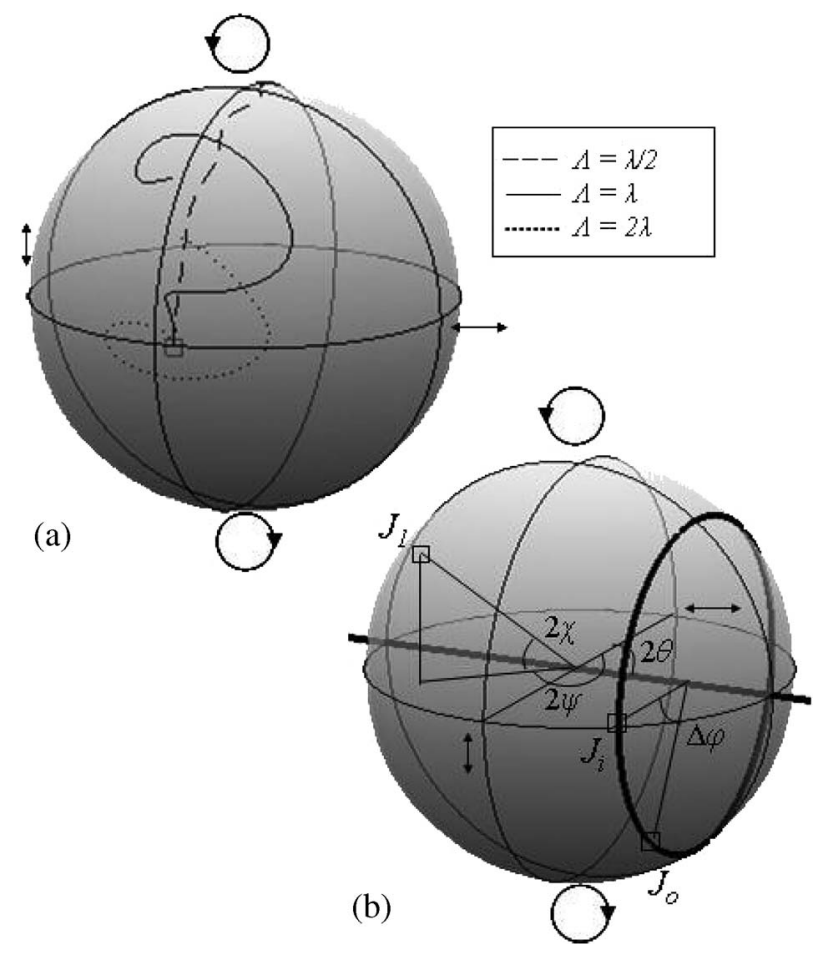

Fig. 5. (a) Poincaré sphere representation of shift in output polarization state for structures with several periods. The input polarization is represented by the square. (b) Performance of an ideal variable wave plate as projected on the Poincaré sphere, where $\theta$ is the optical axis alignment. The input polarization is labeled $J_{i}$, and the output polarization is marked $J_{o}$. Also, the ellipticity $(\chi)$ and azimuth $(\psi)$ are defined for point $J_{1}$.

on the Poincaré sphere for an arbitrary input polarization using phase delay and transmission-efficiency variance results from RCWA. Fig. 5(a) shows the results for structures with an initial depth $\left(d_{0}\right)$ of $3 \lambda$, initial dielectric fill factor $\left(f_{0}\right)$ of 0.5 , $\underline{n}_{2}=1.49$, and several different periods $(\lambda / 2, \lambda$, and $2 \lambda)$. It can be seen at the shorter period that the output polarization state behaves much like an ideal wave plate, as shown in Fig. 5(b). However, as the period increases, the trajectory moves increasingly in a circular fashion. This is the effect of transmissionefficiency variance.

\section{B. Actuation}

The PDMS grating can be deformed in the same manner as a DEA by being placed between two electrodes [Fig. 2(c)]. When a voltage is applied, the rigid electrodes are electrostatically attracted, deforming the grating structure. This compression will decrease the path length and increase the fill factor and effective relative permittivity of the gap between the electrodes, whereas the period remains constant.

The compression of the PDMS structures can be modeled using simple mechanical approximations. The pressure applied on the PDMS structures can be defined as the pressure applied by a parallel-plate capacitor

$$
P=(1 / 2) \varepsilon_{\mathrm{eff}} \varepsilon_{0}\left(\frac{V_{a}}{d+d_{p}}\right)^{2}
$$

where $P$ is the applied pressure, $\varepsilon_{\text {eff }}$ is the effective relative permittivity of the gap between the electrodes, $\varepsilon_{0}$ is the permittivity of free space, $V_{a}$ is the applied voltage, and $d_{p}$ is the underlying PDMS thickness. For simplicity, we assume that the effective relative permittivity remains constant and is the average permittivity of the dielectric material $\left(\varepsilon_{r}\right)$ and of air (1), weighted by their respective volume. By taking into account the Young's modulus and Poisson's ratio and assuming 2-D deformation, the following polynomial equation can be defined [14]:

$$
d^{3}+\left(2 d_{p}-d_{0}\right) d^{2}+\left(d_{p}^{2}-2 d_{p} d_{0}+\frac{\varepsilon_{0} V_{a}^{2} \varepsilon_{\text {eff }}}{2 Y f_{0}}\right) d-d_{p}^{2} d_{0}=0
$$

where $Y$ is the Young's modulus. For this to hold true, several assumptions must be made. The first is that the PDMS will be deformed within a linear Young's modulus region, and that compression will be mostly absorbed by the surface structures, thus changing their fill factor and depth. We must also neglect the effect of the noncompliant electrode on compression, with the top and bottom surfaces of the PDMS structures being "pinned." These assumptions are reasonable if the range of compression is sufficiently small. 


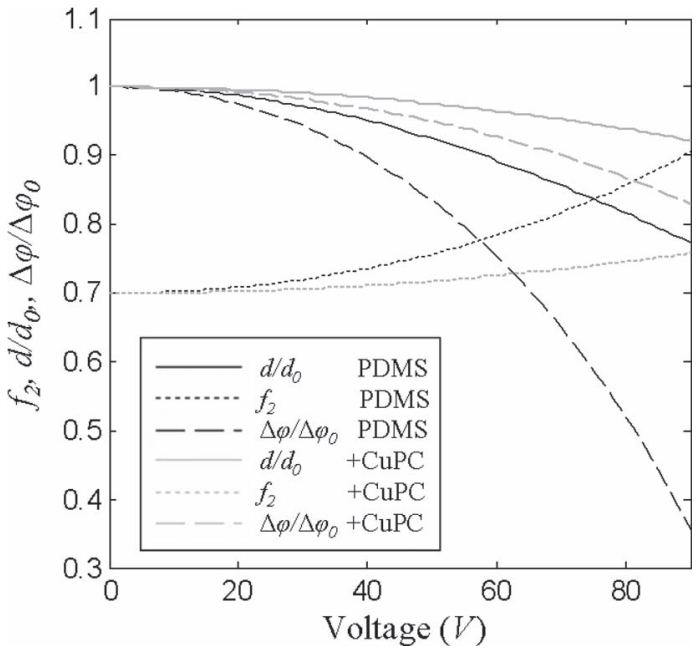

Fig. 6. Fill factor, normalized depth, and normalized polarization phase delay versus applied voltage as calculated using (3) and (4). $d_{0}=1 \mu \mathrm{m}, d_{p}=2 \mu \mathrm{m}$, $\Delta \varphi_{0}=62.9^{\circ}$. Results for both PDMS and PDMS with CuPC additive are shown.

An example of this calculation is shown in Fig. 6, assuming form birefringence $(\Lambda<\lambda)$. In this example, $d_{0}=1 \mu \mathrm{m}$, $d_{p}=2 \mathrm{um}$, and $f_{0}=0.7$. To enhance the performance of the device, a dye is added to the PDMS to increase its dielectric permittivity. Copper phthalocyanine (CuPC), a common dye, is used. This greatly increases the pressure, which can be achieved with the same applied voltage. This method has been previously used to raise the relative permittivity of elastomers to as high as 12 [16]. For our modeling, we estimate a relative permittivity of nine, based on the concentration of CuPC in the PDMS. Fig. 6 shows the modeling results for normal PDMS and PDMS with the CuPC additive.

The effect of this change in geometry through compression is to decrease the birefringence of the structures as the PDMS gradually fills up the gap. We assume that the addition of CuPC has minimal effect on the effective index of refraction of the form-birefringent structures because it is not homogeneously dispersed in the elastomer. While the inhomogeneity of CuPC increased the light scattered off the film and, therefore, the transmission loss (only $1.1 \%$ scattering loss for a typical $10-\mu \mathrm{m}$ film), it was not found to have an adverse effect on the polarization control. This is explained by the observation under microscope at different focal planes that CuPC particles were not present in the grating structures but in the underlying PDMS film. This was further confirmed experimentally by capping a CuPC-doped PDMS grating with CuPC-free PDMS. With the capping layer in place, no diffraction was observed, implying that the grating structures and the capping layer had the same index of refraction. Therefore, the addition of CuPC mainly contributes to the reduction in actuation voltage. This CuPC distribution is accounted for in Fig. 6, where $\varepsilon_{\text {eff }}$ is estimated, assuming that $\mathrm{CuPC}$ is only in the underlying PDMS layer but not in the grating structure.

For ideal performance, the PDMS film should be as thin as possible while maintaining the surface features as deep as possible. This allows for a lower operational voltage and a more efficient transfer of strain into the grating structure while keeping the path length as long as possible. Several similar
PDMS gratings could be stacked to produce an array capable of large polarization phase delays.

\section{DEVICE FABRICATION}

In order to use NIL, it was first necessary to fabricate a rigid master whose pattern could be transferred into the PDMS polymer. Premade photoresist gratings (AZ1512) on silicon were used. Grating 1 had a period of $2 \mu \mathrm{m}$, whereas grating 2 had an asymmetric profile with an overall period of about $20 \mu \mathrm{m}$ (see Fig. 7). Both gratings were square profiled with an initial surface feature depth of $1.3 \mu \mathrm{m}$. The depth of the grating was increased using deep reactive-ion etch (DRIE). Fig. 7(a) and (b) shows scanning electron microscope (SEM) images of these gratings after four- and six-cycle standard Bosch DRIE, respectively. These masters could be used with the photoresist intact but had much greater durability with the photoresist stripped.

Before using a master for PDMS molding, its surface must be made more hydrophobic to allow the release of the hydrophobic cured PDMS. This was done by depositing a monolayer of silane [trichloro $(1 \mathrm{H}, 1 \mathrm{H}, 2 \mathrm{H}, 2 \mathrm{H}$-perfluorooctyl) silane (FOTS)] using vapor-phase deposition.

The PDMS (Dow Sylgard 184) was prepared by mixing PDMS prepolymer with curing agent. The ratio of curing agent to PDMS prepolymer had several effects on the fabrication process and final device. Smaller quantities of curing agent result in softer and stickier PDMS, whereas larger amounts yield a harder and smoother polymer. In view of the final-device operation, a smaller amount of curing agent was desirable because softer PDMS would be easier to compress, thus decreasing the operating voltage. It also allowed for a larger maximum strain and, therefore, larger maximum deformation.

However, using smaller amounts of curing agent led to more "bubbled" surface features which would often stick together. It was found that curing agent ratios as low as $15: 1$ could still be successfully integrated into the fabrication process in place of the more standard $10: 1$ or $5: 1$ ratios.

As mentioned in the previous section, CuPC was added to increase the relative permittivity of the PDMS. This was done by adding $0.5-2.0-w t \%$ copper(II) phthalocyanine from Sigma Aldrich to the PDMS-curing agent mixture. After mixing, the uncured PDMS-curing agent-CuPC mixture was degassed in a vacuum chamber to eliminate air bubbles. The PDMS was molded by depositing a small drop onto the silicon master. A glass-indium-tin oxide (ITO) substrate was then pressed into the PDMS, and pressure was applied using a clamp to flatten the drop into a thin film between the master and the substrate. The PDMS was then cured at $90{ }^{\circ} \mathrm{C}$ for at least $4 \mathrm{~h}$. Higher aspect ratio structures were more successfully transferred with even longer curing times as much as $24 \mathrm{~h}$. The master was then removed, leaving the weakly hydrophobic film of PDMS anchored to the hydrophilic ITO surface. Using this process, a variety of thin PDMS films were made. Fig. 7(c) and (d) shows examples of a thin PDMS film made using gratings 1 and 2 .

The structures in Fig. 7(c) and (d) were formed using the mask in Fig. 8(a) and (b), respectively, with the photoresist stripped. For various master geometries, a small loss in depth was accompanied by a small increase in fill factor between 

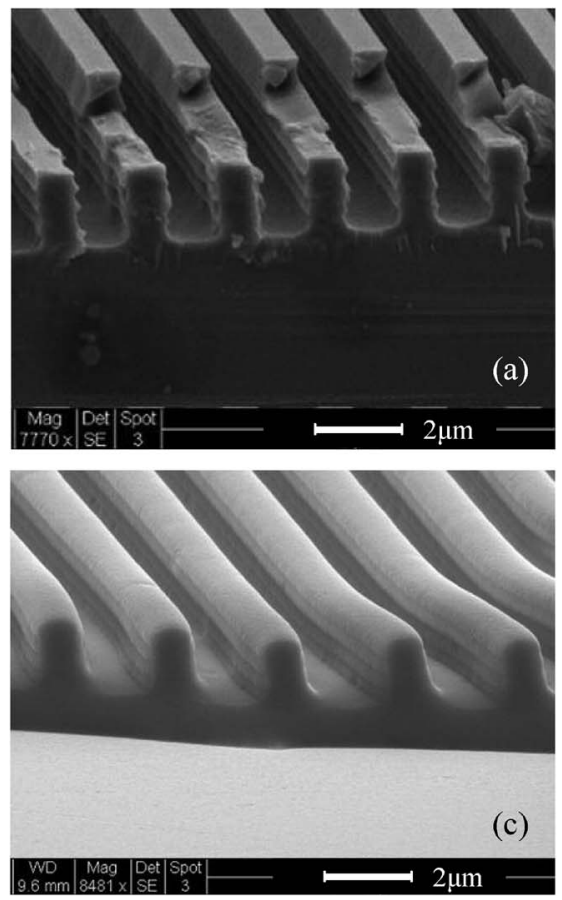
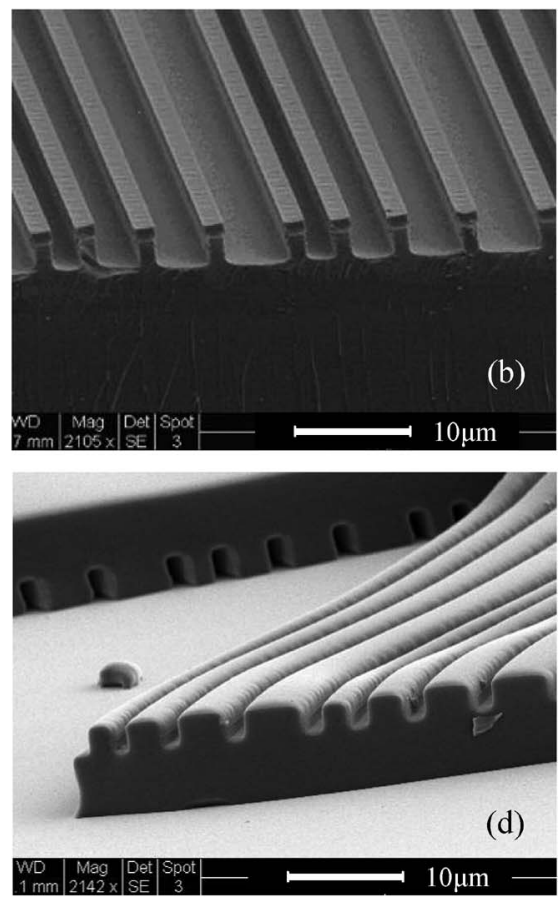

Fig. 7. SEM images of (a) grating-1 master after four-cycle DRIE, (b) grating-2 master after six-cycle DRIE, (c) grating 1 transferred into the PDMS film on ITO, and (d) grating 2 transferred into the PDMS film on ITO.

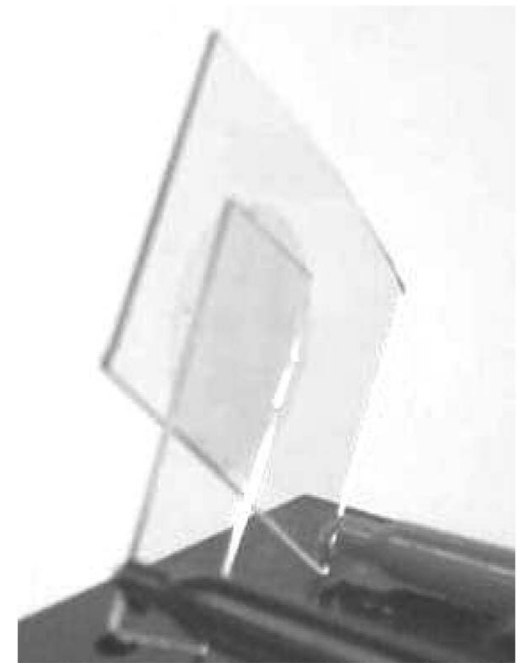

Fig. 8. Photograph of the tunable wave plate made of compressible grating structures, bonded by heating the PDMS in contact with the top ITO-glass plate.

the final film and the original master. This process produced films from 1 to $10 \mu \mathrm{m}$ thick (not including grating structures), depending on the pressure applied between the master and the glass-ITO substrate. The aspect ratio that could be obtained by this method was about 2.5 and was limited by surface features sticking together and the adhesion between the PDMS and ITO (at higher aspect ratios, the adhesion of the PDMS to the deep trenches of the master was greater than the adhesion to the ITO surface of the substrate).

In addition to fabricating thin PDMS films, PDMS blocks were also made. These thick blocks allowed the grating to be compressed by hand to observe their optical properties. These were fabricated by placing the master face-up in a Petri dish and then filling the dish with uncured PDMS. The dish contents were cured for $24 \mathrm{~h}$ at $70^{\circ} \mathrm{C}$. The block was then removed from the dish, peeled off the master, and cured for an additional $1 \mathrm{~h}$ at $90^{\circ} \mathrm{C}$. Cured blocks were approximately $1 \mathrm{~cm}$ thick.

Several methods were attempted for bonding the top glassITO substrate to the patterned PDMS. In the simplest method, the top electrode can be attached by pressing the top glass-ITO onto the PDMS film soon after the master is removed. The tackiness of the PDMS is enough to secure the top plate in place. To strengthen this bond the plates were heated under pressure at $70{ }^{\circ} \mathrm{C}$ for $1 \mathrm{~h}$. Fig. 8 shows this finished device.

\section{Results AND Discussion}

The optical properties of the PDMS gratings were tested using a free-space optical configuration. A 488-nm argon-gas laser was used for a coherent light source. Light was first passed through a rotating linear polarizer so that the incident polarization was linear with a controllable azimuth [see Fig. 5(b) for the description of azimuth and ellipticity]. It was then passed through the grating, and the nondiffracted zeroth-order mode was received by a Thorlabs PAX polarimeter. A voltage source was used for electrostatic compression.

\section{A. Optical Properties}

First, to test the optical properties of the PDMS structures, the large PDMS blocks were used. Being placed between two glass plates, the blocks were placed in the optical path and then squeezed by hand to test the effects of compression.

Surprisingly, the PDMS structures made from grating 2 [i.e., Fig. 7(d)] gave the most consistent and repeatable results despite having an overall period much greater than the 


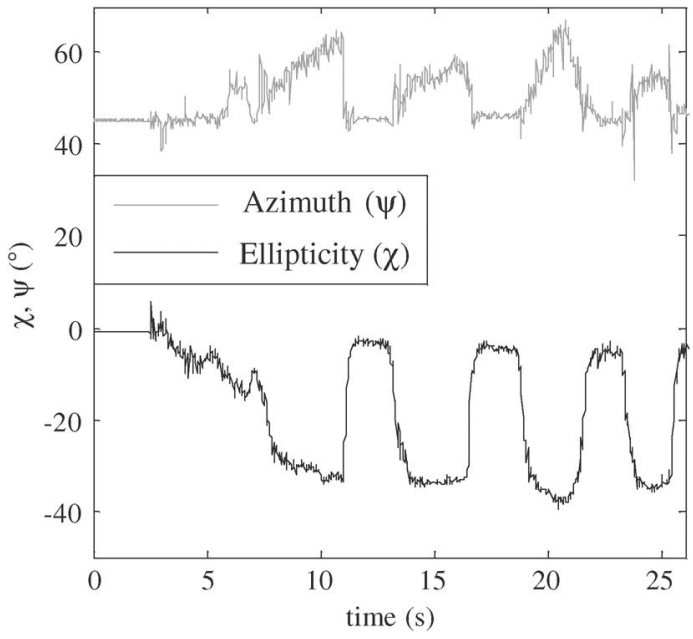

Fig. 9. Output polarization states of a stack of two asymmetric PDMS gratings compressed by hand in the time domain.

wavelength. This allowed multiple gratings to be stacked together for enhanced performance as in the following example. Fig. 9 shows the output polarization states of a parallel stack of two asymmetric PDMS grating blocks being slowly squeezed first (2-10 s) and then squeezed quickly three additional times (14-16, 19-21, and 24-25 s). The grating blocks were aligned visually.

The degree to which a stack of PDMS gratings models ideal wave-plate performance can be seen by testing several different initial polarizations, as was done to create Fig. 10. Regardless of the initial polarization, the output polarization state should be rotated in the same angle around the axis defined on the Poincaré sphere by the alignment of the optical axis of the grating [see Fig. 5(b)]. In the case of Fig. 10, that is the axis connecting vertical and horizontal linear polarization states on the Poincaré sphere. Fig. 10(a) and (b) shows the results for the two stacked grating-2 PDMS blocks. Note in Fig. 10(b) that, for all initial polarization states, the output state is rotated counterclockwise by about $90^{\circ}$ on the Poincaré sphere (the initial states are linearly polarized, that is, on the equator of the Poincaré sphere). Fig. 10(a) shows that, accompanying this rotation around the axis, there is only a slight tilt due to transmissionefficiency variance, which seems to be consistent for all initial polarization states. This result indicates a change in polarization phase delay of $90^{\circ}$, or a variable quarter-wave plate (QWP), for the two stacked asymmetric PDMS gratings. In contrast to the near-ideal performance of grating 2 , grating 1 gave inconsistent results. Although one individual grating block (unstacked) could produce a greater change in polarization than grating 2 , the polarization change was unpredictable and varied from one initial polarization to the next. This is shown in Fig. 10(c).

The performance exhibited by grating 1 , with a period being more than four times the wavelength, can be considered consistent with the results of RCWA, which predicts effects considering both polarization phase delay and transmissionefficiency variance. This leads to the output polarization moving in circles and "diagonal" lines (not rotating about any axis in the linear polarization plane) as the grating is compressed.

The nonideal performance of the grating 1 makes the fact that grating 2, whose overall period is even longer (about $20 \mu \mathrm{m}$ ),

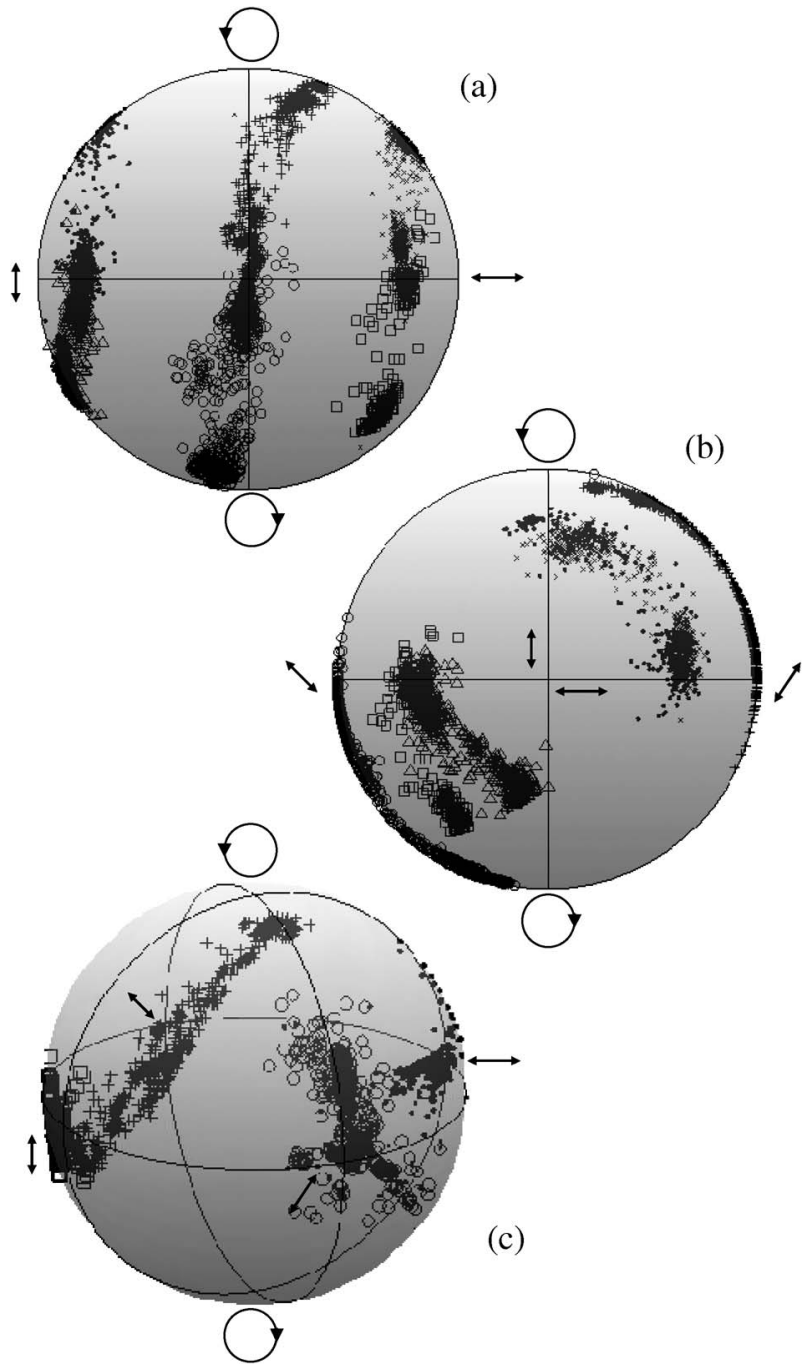

Fig. 10. Output polarization states of $[(a)$ and (b) $]$ a stack of two grating-2 PDMS blocks and (c) one grating-1 PDMS block compressed by hand for different initial linear polarizations. (a) and (b) are the same plots viewed from orthogonal angles on the linear polarization plane.

had such ideal performance even more surprising. By using the same compression model from Section II-B using RCWA instead of form birefringence, the expected performances of gratings 1 and 2 were approximated. To ensure convergence, 80 and 200 diffracted modes were retained in the RCWA model for grating 1 and the longer period grating 2, respectively. Fig. 11 shows the polarization phase delay and transmissionefficiency variance for the two gratings. The geometries were specified to match that of our fabricated PDMS films, namely, $d_{0}=1.8 \mu \mathrm{m}, f_{0}=.4, \Lambda=2 \mu \mathrm{m}$, and $\underline{n}_{2}=1.49$ for grating 1. For grating 2, the profile is shown in Fig. 8(d) with $d_{0}=2.5 \mu \mathrm{m}$ and $\underline{n}_{2}=1.49$.

The results of the RCWA model appear to match the performance of grating 2 in PDMS. First, similar to the experimental results, change in polarization phase delay in the RCWA model is nearly linear with compression. Second and perhaps more importantly, the transmission-efficiency variance for the grating 2 is very small (0.15- $\mathrm{dB}$ maximum ratio change), which is consistent with the smooth rotation on the Poincaré sphere seen in Fig. 10(a) and (b). However, the overall change 


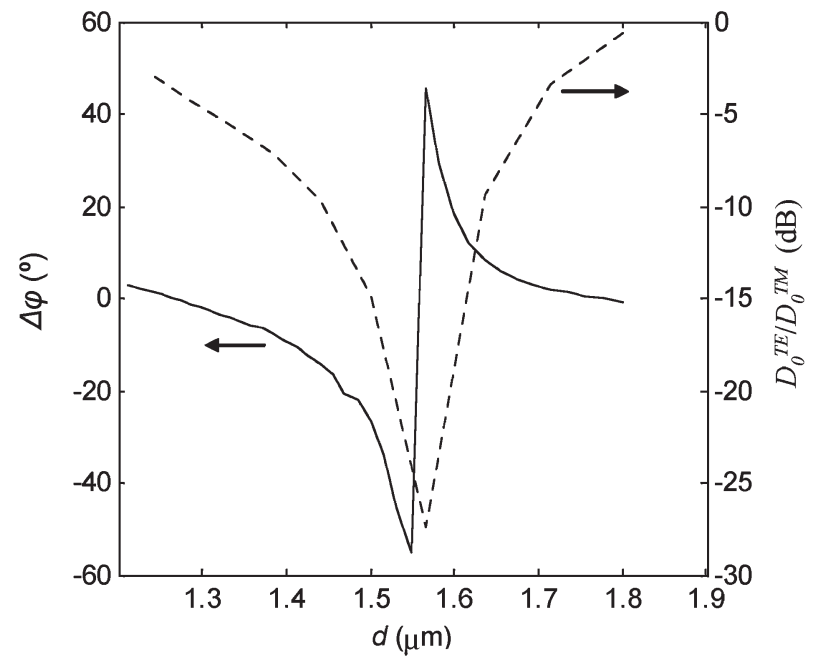

(a)

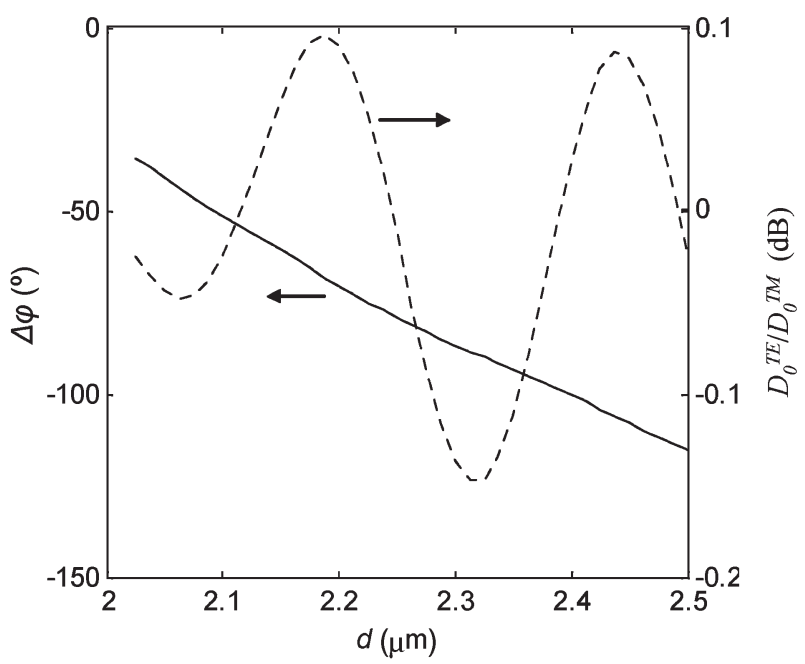

(b)

Fig. 11. Polarization phase delay and transmission-efficiency variance versus compression depth in gratings (a) 1 and (b) 2. Calculations made using RCWA.

in polarization phase delay of the model is significantly larger than the fabricated grating $\left(80^{\circ}\right.$ versus $45^{\circ}$, respectively). This could be due to the assumption of the model that the grating is compressed to $80 \%$ of its original depth, when, in actuality, the deformation may be much less. For example, a polarization phase delay of $45^{\circ}$ would indicate a compression to about $88 \%$ of the original depth.

Compare these results with those for grating 1. As this grating is compressed, it passes through a modal resonance at $d=1.55 \mu \mathrm{m}$, an exact geometry where one polarization mode experiences a large phase delay but poor transmission efficiency. This leads to some large polarization phase delays but also causes large transmission-efficiency variance. This is consistent with the results from Fig. 10(c), where the change of output polarization is relatively unpredictable. However, the full effect of the resonant peak seen in Fig. 11(a) is not observed in the fabricated gratings because an exact square profile is required for the peak to exist as modeled.

From these results, we can infer that the advantage of grating 2 comes from the fact that, with a more complex profile, it avoids modal resonances. In contrast, grating 1 , with a constant period greater than the wavelength of incident light, exhibits the undesirable effects of modal resonance, specifically increased transmission-efficiency variance. Complex geometries are therefore advantageous to gratings which are to act as wave plates because they allow for longer overall periods, thus deeper surface features for the same aspect ratio, while maintaining polarizing properties similar to the form birefringence observed in subwavelength gratings. The disadvantage of the long-period asymmetric grating versus a subwavelength grating is that the long-period grating produces diffraction modes, whereas in the subwavelength grating, all power is transferred to the zeroth-order mode only. Transmission efficiencies to the zeroth-order mode for gratings 1 and 2 were 0.25 and 0.39 per layer, respectively. Diffracted modes could potentially interfere with the performance of an integrated device.

\section{B. Device Performance}

The final performance of the device was tested by measuring the change in phase delay through the tunable wave plate under applied voltage. Many devices were fabricated and tested with different combinations of curing agent ratios, curing pressures (determining film thickness), and top-plate bonding methods. Most devices were found to exhibit little or no change in polarization phase delay. This was assumed to be due to inefficient compression of the PDMS structures.

The first problem decreasing the effectiveness of compression was areas of the grating being filled solid. The film relies on the surface structures to be able to properly compress. Small areas of solid PDMS between the electrodes prevented the entire grating area from compressing properly.

Another problem affecting compression was that direct heat bonding was much more effective with thicker films. This is because thicker films $(>10 \mu \mathrm{m})$ allowed for more conformal contact between the PDMS and ITO. Thinner films resulted in very weak bonds that usually broke during testing. Therefore, in order to create a more durable device, the spacing between the plates had to be increased, thus increasing the required voltage.

Despite the previously mentioned setbacks in the final stage of fabrication, devices were realized, which could produce significant polarization phase delays although reduced from those observed with the thick PDMS blocks under manual compression. This reduction could be due to either insufficient applied voltage to observe the full range of PDMS compression or slight differences in the PDMS structure geometries between the blocks and the films due to the different curing pressures used in the two processes. Fig. 12 shows the voltage-to-phasedelay relation of a device fabricated using the grating-1 master, a 10:1 curing agent ratio, low curing pressure, and direct heat bonding. A total change in polarization phase delay greater than $10^{\circ}$ was observed. While the total change in polarization phase delay is reduced, the shape of the curve in Fig. 12 matches the expected performance predicted by the theoretical modeling using form birefringence, as shown in Fig. 6. Note that the magnitude of the slope of the curve increases with voltage, as predicted by the model. 


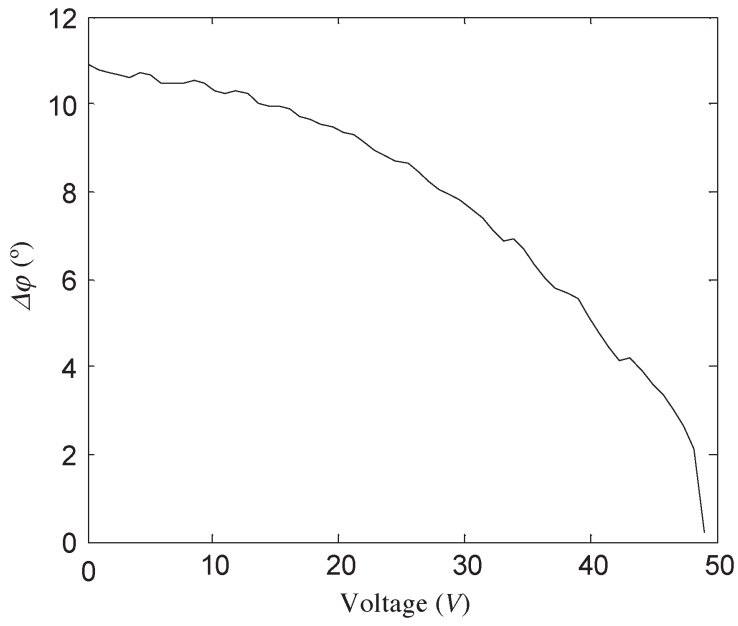

Fig. 12. Polarization phase delay versus applied voltage for the fabricated device.

\section{CONCLUSION}

A compact and low-cost design for a variable wave plate using tunable form-birefringent structures in PDMS was proposed, modeled, and fabricated. The optical properties of the PDMS structures were verified using manually compressed blocks. The polarization tuning properties of the asymmetric grating-2 profile proved nearly ideal, and using two stacked gratings, a variable QWP was achieved. The use of complex profile gratings for polarization applications presents an alternative to subwavelength form-birefringent structures. The use of complex profile structures allows for longer periods, deeper surface features (greater path lengths), and lower aspect ratios while maintaining satisfactory polarizing tuning performance.

There is still room for improvement in the optical properties and actuation of the device. The integrated device performance could be enhanced by several improvements. First, the capacity to increase the index of refraction of the PDMS would allow for much greater polarization effects with less compression. CuPC proved very difficult to disperse in the PDMS to the degree that it would improve the optical properties. Second, more effective bonding methods could decrease the spacing between the electrode plates, thus increasing the compression achieved by the same voltage.

\section{ACKNOWLEDGMENT}

The authors would like to thank the help from both the NanoTech User Facility, University of Washington, Seattle, and the Washington Technology Center for the help with fabrication.

\section{REFERENCES}

[1] W. Nakagawa and H. P. Herzig, "Adjustable waveplate based on formbirefringent nanostructures," in Proc. IEEE Int. Conf. Optic. MEMS Appl., 2004, pp. 202-203.

[2] H. Kikuta, H. Toyota, W. Yu, A. Mizutani, and K. Iwata, "Optical elements with subwavelength structured surfaces," Proc. SPIE, vol. 5515, pp. 125131, 2004.

[3] T. Isano, Y. Kaneda, N. Iwakami, K. Ishizuka, and N. Suzuki, "Fabrication of half-wave plates with subwavelength structures," Jpn. J. Appl. Phys. 1, Regul. Rap. Short Notes, vol. 43, no. 8A, pp. 5294-5296, Aug. 2004.
[4] M. P. Nezhad, C. Tsai, L. Pang, W. Nakagawa, G. Klemens, and Y. Fainman, "Form birefringent retardation plates in GaAs substrates: Design, fabrication and characterization," Proc. SPIE, vol. 5225, p. 69, 2003.

[5] S. Uma, R. Matusiak, D. L. Hecht, and E. J. Shrader, "Electrostrictive elastomer based diffractive modulator for use as a variable optical attenuator," IEEE J. Sel. Topics Quantum Electron., vol. 10, no. 3, pp. 435-439, May/Jun. 2004.

[6] Y.-C. Tung and K. Kurabayashi, "Nanoimprinted strain-controlled elastomeric gratings for optical wavelength tuning," Appl. Phys. Lett., vol. 86, no. 16, p. 161113 , Apr. 2005.

[7] M. Aschwanden and A. Stemmer, "Polymeric, electrically tunable diffraction grating based on artificial muscles," Opt. Lett., vol. 31, no. 17, pp. 2610-2612, Sep. 2006.

[8] R. Kornbluh, R. Pelrine, H. Prahlad, and R. Heydt, "Electroactive polymers: An emerging technology for MEMS," Proc. SPIE, vol. 5344, pp. 13-27, 2004.

[9] P. Sommer-Larsen and A.-L. Larsen, "Materials for dielectric elastomer actuators," Proc. SPIE, vol. 5385, pp. 68-77, Jul. 2004.

[10] B. Grzybowski, D. Qin, R. Haag, and G. M. Whitesides, "Elastomeric optical elements with deformable surface topographies: Applications to force measurements, tunable light transmission and light focusing," Sens. Actuators, vol. 6, no. 1/2, pp. 81-85, 2000.

[11] K. H. Jeong, G. L. Liu, N. Chronis, and L. P. Lee, "Tunable microdoublet lens array," Opt. Express, vol. 12, no. 11, pp. 2494-2500, May 2004.

[12] V. J. Cadarso, A. Llobera, G. Villanueva, C. Domínguez, and J. A. Plaza, "3-D modulable PDMS-based microlens system," Opt. Express, vol. 16, no. 7, pp. 4918-4929, Mar. 2008.

[13] M. Born and J. Wolf, Principles of Optics: Electromagnetic Theory of Propagation, Interference and Diffraction of Light, vol. 6. Cambridge, U.K.: Cambridge Univ. Press, 1999, pp. 837-840.

[14] M. G. Moharam and T. K. Gaylord, "Diffraction analysis of dielectric surface-relief gratings," J. Opt. Soc. Amer, vol. 72, no. 10, pp. 1385-1392, Oct. 1982.

[15] M. G. Moharam, E. B. Grann, D. A. Pommet, and T. K. Gaylord, "Formulation for stable and efficient implementation of the rigorous coupledwave analysis of binary gratings," J. Optic. Soc. Amer. A, Opt. Image Sci., vol. 12, no. 5, pp. 1068-1076, May 1995.

[16] X. Zhang, C. Lowe, M. Wissler, B. Jahne, and G. Kovacs, "Dielectric elastomers in actuator technology," Adv. Eng. Mater, vol. 7, no. 5, pp. 361367, May 2005.

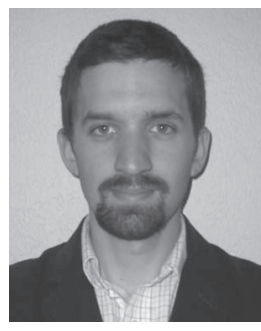

Benjamin K. Wilson received the B.S. and M.S. degrees in electrical engineering from the University of Washington, Seattle, in 2005 and 2007, respectively, where he is currently working toward the Ph.D. degree.

$\mathrm{He}$ is currently a Research Assistant with the Department of Electrical Engineering Photonics Laboratory, University of Washington. His research interests include nanophotonics, biophotonics, and optical MEMS integration.

Lih Y. Lin (M'94-SM'02) received the Ph.D. degree in electrical engineering from the University of California, Los Angeles, in 1996.

She was then with AT\&T Labs-Research as a Senior Technical Staff Member, working on micromachined technologies for optical switching and lightwave systems. In 2000, she was with Tellium as Director of Optical Technologies. She has been an Associate Professor with the Department of Electrical Engineering, University of Washington, Seattle, since 2003. She has over 170 publications in refereed journals and conference proceedings. She is the holder of 22 U.S. patents.

Dr. Lin is a member of the Optical Society of America. She has served on the technical program committees of and cochaired various technical conferences. Currently, she is on the Steering Committee of the International Conference on Optical MEMS and Nanophotonics. She was the Guest Editor for the IEEE Journal of SELECTED TOPICS IN QUANTUM ELECTRONICS Special Issue on Optical Micro- and Nanosystems and the IEEE/OSA JOURNAL OF Lightwave TeChnology Special Issue on Optical MEMS and Its Future Trends. She received the MIT Technology Review 100 Award in 2003. 